\title{
Metatarsal Salmonella enteritidis osteomyelitis in a healthy child
}

\author{
Sara Limão, Tânia Carvalho, Helena Sousa, Florbela Cunha
}

Pediatrics, Hospital Vila Franca de Xira, Lisbon, Portugal

\section{Correspondence to \\ Dr Sara Limão,}

saralimao@netcabo.pt

Accepted 1 May 2019

Check for updates

(C) BMJ Publishing Group Limited 2019. No commercial re-use. See rights and permissions. Published by BMJ.

To cite: Limão S, Carvalho T, Sousa $\mathrm{H}$, et al. BMJ Case Rep 2019;12:e227765. doi:10.1136/bcr-2018227765

\section{SUMMARY}

Non-typhoidal Salmonella osteomyelitis in healthy children is a very rare condition. A previously healthy 7-year-old boy presented with foot pain following a small injury. Local inflammatory signs of the foot were observed, with a normal radiograph. Two weeks before, he had had gastroenteritis. Assuming cellulitis, flucloxacillin was began. Early during admission, surgical subcutaneous abscess drainage was performed and Salmonella enteritidis was identified. According to bacterial susceptibility, antibiotherapy was changed to ceftriaxone. A foot radiograph and an MRI scan, performed on the 9th and the 12th days, revealed findings suggestive of osteomyelitis of the metaphysis of the fifth metatarsal. Clinical worsening persisted and surgical extensive debridement was needed. A 6-week antibiotic treatment was completed, with a good outcome. Osteomyelitis with a poor evolution in children with risk factors (prior gastrointestinal illness, warm weather and previous exposure to antibiotics) can raise the possibility of a non-typhoidal Salmonella infection.

\section{BACKGROUND}

Acute hematogenous osteomyelitis (AHO) is the most common type of bone infection in children and is usually due to Staphylococcus aureus. It characteristically affects the metaphysis of the long bones, where tortuous blood flow promotes bacteria aggregation. The infection is more common in the lower extremities and generally involves a single bone. ${ }^{12}$ Metatarsal osteomyelitis is rare, accounting for $1 \%-2 \%$ of all the cases in children. ${ }^{3}$ Boys are more affected, in an estimated rate of $2: 1 .^{23}$

Salmonella is a common cause of gastroenteritis, but Salmonella osteomyelitis is rare, complicating an estimated $0.8 \%$ of Salmonella infections and constituting about $0.45 \%$ of all types of osteomyelitis. ${ }^{4}$

Most cases of Salmonella osteomyelitis occur in individuals with immunodeficiencies or haemoglobinopathies, mainly sickle cell disease (SCD), but are very rare in healthy children. ${ }^{4-9}$ Non-typhoidal Salmonella (NTS) strains are the most commonly implicated, namely Salmonella enteritidis, Salmonella typhimurium and Salmonella paratyphi B. ${ }^{78}$ Salmonella enteritidis is responsible for up to $85 \%$ of cases of NTS bacteraemia in children.?

Salmonella osteomyelitis has no specific clinical features although it tends to cause more severe clinical syndromes. As with other pathogens, the bone infection can evolve to an intraosseous or a subperiosteal abscess, or a contiguous septic arthritis.
The authors describe this case due to its rarity and diagnostic challenges.

\section{CASE PRESENTATION}

A previously healthy caucasian 7-year-old boy, with no relevant family history, presented during the summer to the paediatric emergency department (PED) with left foot pain following an injury caused by an impact of a football on the previous day. Physical examination revealed pain, heat, redness and slight swelling in the anterior half of the external border of left foot, with a small excoriation. Mobility of ankle and foot was preserved, and there were no abnormal neurovascular signs. A diagnosis of cellulitis was assumed, and he was treated with empirical oral antibiotic therapy (flucloxacillin).

He returned to the PED 2 days after with worsening pain and local inflammatory signs, along with limitation of ankle movements, and was hospitalised for intravenous antibiotherapy with amoxicillin/clavulanic acid and clindamycin. There was no fever, or other systemic signs or symptoms.

Three weeks before admission, he had had an acute tonsillitis, treated with intramuscular benzylpenicillin, and a week later, a self-limited febrile gastroenteritis.

There was no history of contact with reptiles, recent foreign travel or consumption of undercooked poultry, eggs or dairy products.

After hospital admission, there was a worsening of the inflammatory signs of the distal portion of the external left foot border, with extension to the fifth toe, accompanied with elevation of acutephase proteins. On the third day, superficial surgical exploration was performed with drainage of pus, which was collected for bacterial culture.

After incision and drainage, a slight improvement of local inflammatory signs was observed, but increased pain on palpation or movement. Bacterial culture revealed Salmonella enteritidis, and based on antibiotic sensitivities antibiotherapy was changed to ceftriaxone. The patient remained afebrile, however, he had mild anorexia and adynamia and there was poor wound healing.

On the 16th day of inpatient admission, signs of local inflammation worsened, with a fluctuant swelling on palpation. A deep surgical wound exploration was performed, finding bone destruction, sequestra and septic arthritis of the fifth metatarsophalangeal joint. 


\section{INVESTIGATIONS}

Laboratory tests on admission revealed haemoglobin of $12.60 \mathrm{~g} /$ $\mathrm{dL}$, C-reactive protein (CRP) of $5.37 \mathrm{mg} / \mathrm{dL}$ (reference range $0.06-1.00 \mathrm{mg} / \mathrm{dL}$ ), white blood cell count (WBC) of 13 900/ $\mathrm{mm}^{3}$ and neutrophils of $7260 / \mathrm{mm}^{3}$. The CRP, WBC and neutrophils continued to rise until the third day of admission, with the CRP reaching $12.00 \mathrm{mg} / \mathrm{dL}$. Subsequently, CRP level and WBC progressively decreased until normal levels. On the 12th day, CRP was normal $(<0.50 \mathrm{mg} / \mathrm{dL})$ and erythrocyte sedimentation rate (ESR) was $30 \mathrm{~mm} /$ hour.

Salmonella enteritidis was identified from the cultures of pus (collected on days 3 and 16) and was susceptible to ampicillin, third-generation cephalosporins and carbapenems.

Blood and stool cultures were sterile.

Immunological laboratory tests including HIV, immunoglobulins, $\mathrm{CD}^{+}{ }^{+}$and $\mathrm{CD} 8^{+} \mathrm{T}$ cells and complement were normal. Interleukin-12/interferon-gamma (IL-2/IFN- $\gamma$ ) axis and neutrophil oxidative burst assay were normal. A peripheral blood smear did not reveal alterations. An abdominal ultrasound was performed, showing a normal spleen and no other alterations. Splenic scintigraphy to rule out functional hyposplenism was not performed.

Left foot radiographs taken at the first visit to the PED and on the third day were normal. On day 9, a foot radiograph revealed an osteolytic lesion of the distal metaphysis of the fifth metatarsal bone (figure 1).

A skin and soft tissue ultrasound was performed on the second and eighth days, showing subcutaneous oedema with no abscess.

An MRI (without contrast) performed on the 12th day showed signs of acute osteomyelitis of the distal diaphysis and metaphysis of the fifth metatarsus, along with medullary and adjacent subcutaneous oedema, without cloaca, abscess formation and sequestra. Sequestra was subsequently identified on day 16 at the time of surgical debridement.

\section{DIFFERENTIAL DIAGNOSIS}

The initial diagnosis that was assumed was cellulitis because the child presented with left foot inflammatory signs and a small excoriation. As symptoms and laboratory acute-phase reactants continued to worsen after 48 hours of antibiotics, other diagnosis were considered. In a previously healthy child with acute local pain, limited movement and local inflammatory signs, the differential diagnosis includes osteomyelitis and septic arthritis, which can occur without fever. Bone tumours are less probable in the presence of acute symptoms without constitutional symptoms, lymphadenopathy or organomegaly.

Arthritis and tumours were excluded by imaging evaluation. Radiological signs of osteomyelitis, which are normally delayed, were present on day 9, and subsequently identified on MRI.

The most probable aetiology of osteomyelitis in a healthy immunocompetent child is Staphylococcus aureus. In the presented case, isolation of Salmonella enteritidis from pus confirmed the unexpected diagnosis of NTS osteomyelitis.

\section{TREATMENT}

After the first visit to the PED, the patient was discharged on oral flucloxacillin.

In the inpatient setting, the child was medicated with intravenous amoxicillin/clavulanic acid and clindamycin.

Subcutaneous abscess drainage was performed on day 3, and treatment with intravenous ceftriaxone, after obtaining antibiotic sensitivity, was initiated on the 9th day.

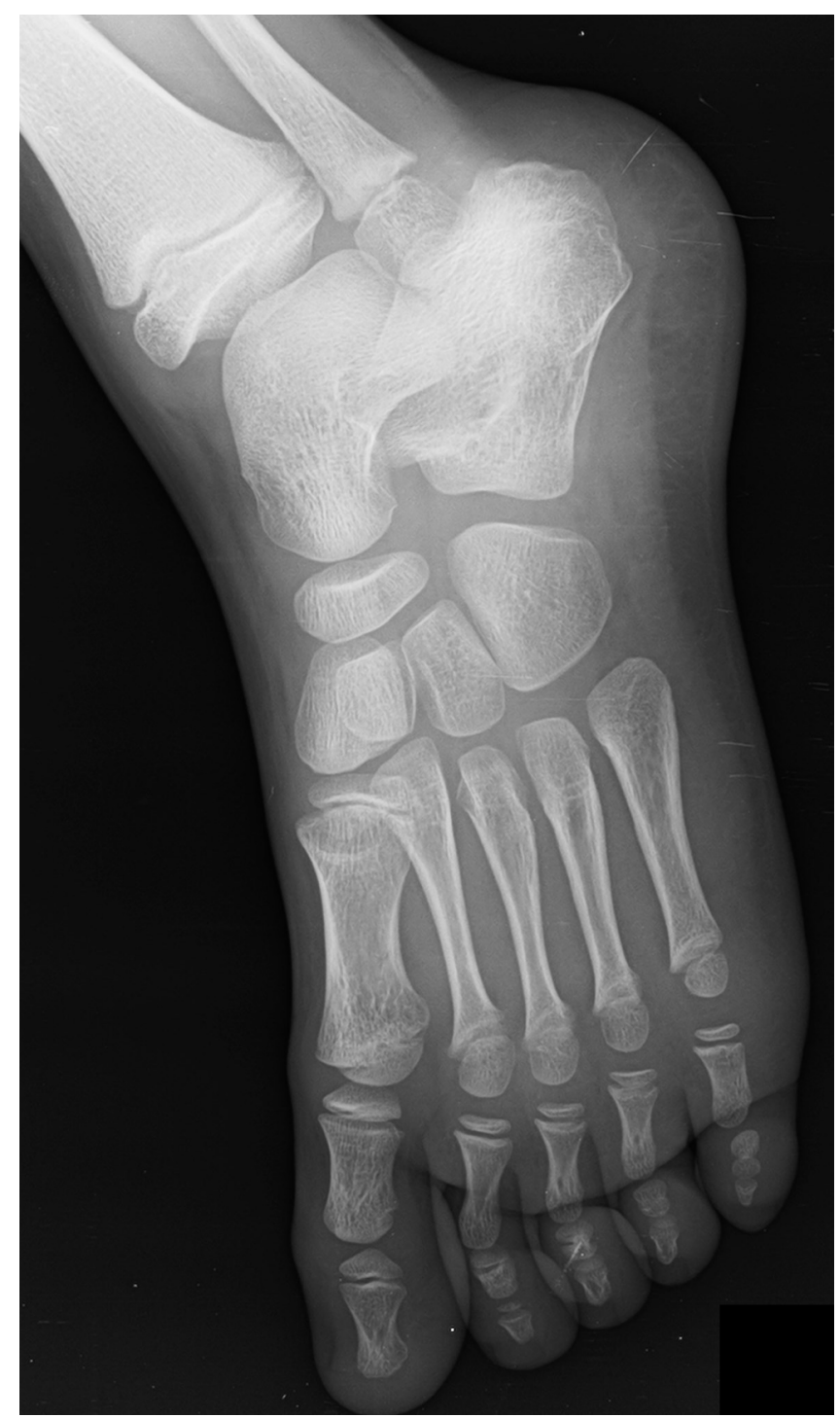

Figure 1 Left foot radiograph on day 9 of admission showing an osteolytic lesion of the distal metaphysis of the fifth metatarsal bone.

On the 16th day, surgical extensive debridement was performed, with removal of necrotic tissue and sequestra of the distal epiphysis of the fifth metatarsal and the adjacent portion of the first phalange.

The patient completed a 4-week treatment with intravenous ceftriaxone, along with physiotherapy, followed by oral treatment with amoxicillin for an additional period of 2 weeks, as an outpatient.

\section{OUTCOME AND FOLLOW-UP}

The patient was discharged with near-total functional recovery. He maintained physiotherapy during 4 months, with full functional recovery. A radiograph taken 3 months after discharge showed mild shortening of the distal portion of the fifth metatarsal. The child continues in outpatient paediatric and orthopaedic follow-up, and in the 1-year period post discharge there were no further complications.

\section{DISCUSSION}

Salmonella osteomyelitis is more common in children with SCD, primary immunodeficiencies (defects of the IL- $2 / \mathrm{IFN}-\gamma$ axis) 
and acquired immunodeficiencies (HIV, malignancy, immunosuppressive therapy). ${ }^{4-7} 9-11$ In otherwise healthy children, it is very rarely reported. ${ }^{4-9} \mathrm{~A}$ recent review shows 46 cases of osteomyelitis due to NTS in children without a known immunodeficiency or SCD between 1960 and 2016. ${ }^{7}$ The most commonly affected bones are the humerus and vertebrae, followed by the tibia and pelvis. ${ }^{4}$ The metatarsals have been extremely rarely implicated in Salmonella osteomyelitis. ${ }^{12}$ The presented case describes a previously healthy child with metatarsal Salmonella osteomyelitis, whose immunological and haematological evaluation was normal.

Previous gastrointestinal illness, warm weather and pre-exposure to antibiotics have been described as risk factors for the development of NTS bacteraemia in several studies. ${ }^{10}$ Antibiotics active against anaerobic species, such as penicillin, may increase the risk by reducing the protective anaerobic gut flora. ${ }^{10}$ The authors believe that a previous gastroenteritis, together with a recent antibiotic exposure, may have triggered a transient bacteraemia in this patient. It is also possible that the previous minor local trauma contributed to the infection, since this is known to be a risk factor for AHO. ${ }^{1}$ Other classic risk factors for Salmonella infection, including exposure to reptiles, recent foreign travel and exposure to undercooked poultry, eggs or dairy products, were identified in only 14\% of NTS osteomyelitis cases (6/42).

The diagnosis of $\mathrm{AHO}$ is difficult and often delayed, as the radiographic abnormalities take about $10-14$ days to emerge, ${ }^{1-3}$ because lytic lesions require at least $50 \%$ of the mineral density to be depleted. ${ }^{2}$ A radiograph is usually the initial imaging study and allows exclusion of other conditions, but has low sensitivity and specificity for AHO. In this case, a lytic lesion was detected on the 12th day of symptoms (the 9th day of admission).

MRI is the most sensitive imaging exam in detecting earlier signs of osteomyelitis, ${ }^{129}$ such as marrow oedema, which is present in early stages. ${ }^{1}$ Evidence suggests that earlier MRI leads to a reduced hospital stay. ${ }^{2}$

No specific laboratory tests for AHO are available. Non-specific serum markers of inflammation, mainly WBC and CRP, may be a useful adjunct to establish the diagnosis and monitor response to therapy. ${ }^{1-3}$ CRP is more useful than ESR as it rises earlier and normalises more quickly after institution of the effective therapy. ${ }^{13}$

The definitive diagnosis is made when a pathogen is isolated from local tissue or pus. In this case, Salmonella enteritidis was identified. A recent review of NTS osteomyelitis in otherwise healthy children reports positive blood cultures in only 10 of 28 cases $(36 \%),{ }^{7}$ and a previous literature review reveals even a lower percentage of positive results $(15 \%) .^{5}$

Clinicians must have a high index of suspicion for this condition. This case highlights that AHO can develop with no systemic manifestations such as fever. A literature review of NTS osteomyelitis in apparently healthy children revealed that about $55 \%$ of the patients $(11 / 20)$ had fever at the onset of illness, while almost all presented with local pain, swelling or both. ${ }^{5}$ The preceding bacteraemia leading to AHO in children is usually transitory and asymptomatic. The clinical hallmark of osteomyelitis is localised pain and reduced function, which were the red flags in our patient. ${ }^{1}$

Management of children with AHO demands a multidisciplinary approach. Although large randomised controlled trials are scarce, there are published guidelines for the treatment of osteoarticular infections in children. ${ }^{13}$ Early empiric parenteral antibiotherapy directed against methicillin-sensitive Staphylococcus aureus is advised. ${ }^{12}{ }^{13}$ Antibiotics of choice are beta-lactams, namely first-generation or second-generation cephalosporins. Clindamycin should be chosen in settings with a prevalence of methicillin-resistant Staphylococcus aureus (MRSA) higher than 10\%-15\%. ${ }^{13}$ The child's age and the circumstances around the onset of the infection may give a clue to other possible causative organisms. Regional bacterial susceptibility patterns should also be considered. If antibiotic sensitivity data are known, subsequent therapy should be adjusted. If possible, cultures (bone, synovial or abscess fluid) should be obtained. Radiographic resolution is delayed, and therefore, should not be used to guide treatment. ${ }^{1}$ Antibiotic treatment in AHO should be maintained for a minimum of 3-4 weeks in healthy children $>3$ months, but in complicated cases or those caused by unusual or resistant agents such as Salmonella and MRSA, a longer duration of total antibiotherapy is advised (up to 6 weeks in some centres). ${ }^{13}$ Early switching from intravenous to oral antibiotherapy is recommended for uncomplicated AHO with clinical and laboratory improvement, but a more prolonged intravenous therapy is advised in the presence of virulent pathogens such as Salmonella. ${ }^{13}$

Antimicrobial resistance of NTS species is rising worldwide, with geographic differences. In North America, an increase in clinically important resistance to ampicillin, ceftriaxone, ciprofloxacin, gentamicin and trimethoprim-sulfamethoxazole has been reported. ${ }^{14}$ Furthermore, NTS resistance to fluoroquinolones and third-generation cefalosporins is increasing. Azithromycin and carbapenems are possible alternatives, as resistance to these agents has rarely been noted. ${ }^{15}$

Surgery has no advantage over conservative treatment in acute osteomyelitis, even though it should be considered in cases with poor response to antibiotherapy within $48-72$ hours, abscess formation or other suspected complications. ${ }^{1-38} 813$ In a review of 13 cases of NTS osteomyelitis in healthy children, about half were treated with a combination of surgical debridement and antibiotherapy, ${ }^{4}$ showing that osteomyelitis caused by this agent often requires surgery.

Complications of AHO may include chronic infection, septic arthritis, avascular necrosis, growth disturbance, pathologic fractures and sepsis, among others. In the presented case, although targeted antibiotherapy was administered, the inflammatory process evolved to bone destruction and sequestra formation, which was identified and treated only by a surgical procedure.

Most authors recommend treatment of chronic Salmonella osteomyelitis with surgical debridement and drainage combined

\section{Learning points}

- Salmonella osteomyelitis in otherwise healthy children is an extremely rare and serious condition, mainly caused by nontyphoidal strains.

- Previous gastrointestinal illness, warm weather and preexposure to antibiotics have been described as risk factors for non-typhoidal Salmonella bacteraemia.

- NTS osteomyelitis has no specific clinical features, laboratory or imaging signs compared with osteomyelitis caused by other agents, but the rate of complications and poor outcome appears to be higher.

- Blood cultures are usually sterile; cultures of pus should be obtained if possible, especially if there is poor response to empiric antibiotherapy, as directed treatment is crucial for a good outcome. 
with prolonged antibiotherapy, with no consensus on the duration of therapy. ${ }^{4}$

Although an infrequent condition, evidence shows that NTS osteomyelitis in previously healthy children has an important rate of complications. Abscess formation was described in 11 out of 46 cases $(24 \%) .^{7}$ The recurrence rate in healthy children appears to be low, ${ }^{4}$ although higher than for Staphylococcus aureus osteomyelitis. ${ }^{7}$ Therefore, follow-up is advised. Nevertheless, prognosis of NTS osteomyelitis needs further investigation.

Acknowledgements The authors thank Dr Clara Júlio for her contribution to the diagnostic assessment and surgical management of the patient; and Dr Merlin McMillan for his contribution to improving writing.

Contributors SL, TC, HS and FC have substantially contributed to the conception or design of the work, or the acquisition, analysis or interpretation of data for the work; drafting the work or revising it critically for important intellectual content; final approval of the version to be published; and agreement to be accountable for all aspects of the work in ensuring that questions related to the accuracy or integrity of any part of the work are appropriately investigated and resolved.

Funding The authors have not declared a specific grant for this research from any funding agency in the public, commercial or not-for-profit sectors.

Competing interests None declared.

Patient consent for publication Parental/guardian consent obtained.

Provenance and peer review Not commissioned; externally peer reviewed.

\section{REFERENCES}

1 Conrad DA. Acute hematogenous osteomyelitis. Pediatr Rev 2010;31:464-71.
2 Funk SS, Copley LA. Acute hematogenous osteomyelitis in children: pathogenesis, diagnosis, and treatment. Orthop Clin North Am 2017;48:199-208.

3 Peltola H, Pääkkönen M. Acute osteomyelitis in children. N Engl J Med 2014:370:352-60.

4 Tsagris V, Vliora C, Mihelarakis I, et al. Salmonella osteomyelitis in previously healthy children: report of 4 cases and review of the literature. Pediatr Infect Dis J 2016;35:116-7.

5 Dutta A, Allen CH. Non-typhoidal Salmonella osteomyelitis in the midfoot of a healthy child and review of the literature. J Infect Dis Ther 2013;1:107.

6 Balakumar B, Gangadharan S, Ponmudi N, et al. Atypical osteomyelitis and concurrent septic arthritis due to Salmonella in immunocompetent children. J Clin Orthop Trauma 2017:8:293-7.

7 Gill AN, Muller ML, Pavlik DF, et al. Nontyphoidal Salmonella osteomyelitis in immunocompetent children without hemoglobinopathies: a case series and systematic review of the literature. Pediatr Infect Dis J 2017;36:910-2.

8 Abdulla M, Khaled YS, Kapoor H. Salmonella osteomyelitis of the calcaneum bone in an immunocompetent child. J Pediatr Orthop B 2014;23:270-3.

9 Kung YH, Liang JL, Che Kuo K, Kuo KC. Non-typhoidal Salmonella osteomyelitis of the femoral neck in an immunocompetent child. J Arthritis 2017;06:241.

10 Grisaru-Soen G, Wysoki MG, Keller N. Risk factors for development of nontyphoid Salmonella bacteremia. Clin Pediatr 2004:43:825-9.

11 Meyyur Aravamudan V, Kee Fong P, Singh P, et al. Extraintestinal Salmonellosis in the Immunocompromised: An Unusual Case of Pyomyositis. Case Rep Med 2017;2017:1-5.

12 Mackenjee MK, Coovadia HM, Naidoo LS, et al. Salmonella osteomyelitis. S Afr Med J 1974;48:591-2.

13 Saavedra-Lozano J, Falup-Pecurariu O, Faust SN, et al. Bone and Joint Infections. Pediatr Infect Dis J 2017;36:788-99.

14 MacFadden DR, Bogoch II, Andrews JR. Advances in diagnosis, treatment, and prevention of invasive Salmonella infections. Curr Opin Infect Dis 2016;29:453-8.

15 Hohmann EL. Nontyphoidal Salmonella bacteremia. 2019 https://www.uptodate.com/ contents/nontyphoidal-salmonella-bacteremia (Accessed on 13th Jan 2019).

Copyright 2019 BMJ Publishing Group. All rights reserved. For permission to reuse any of this content visit

https://www.bmj.com/company/products-services/rights-and-licensing/permissions/

BMJ Case Report Fellows may re-use this article for personal use and teaching without any further permission.

Become a Fellow of BMJ Case Reports today and you can:

- Submit as many cases as you like

- Enjoy fast sympathetic peer review and rapid publication of accepted articles

- Access all the published articles

- Re-use any of the published material for personal use and teaching without further permission

For information on Institutional Fellowships contact consortiasales@bmjgroup.com

Visit casereports.bmj.com for more articles like this and to become a Fellow 